FC risk factors may have an affinity to the right hemisphere. (Janszky J, Woermann FG, Barsi P et al. Neurology April 8, 2003;60:1209-1210).

The pathological basis of temporal lobe epilepsy (TLE) was studied in 22 children undergoing temporal lobectomy for refractory seizures at Hopital Ste-Justine, Universite de Montreal, Canada (Bocti C, Robitaille Y, Diadori P, et al. Neurology Jan (2 of 2) 2003;60:191-195). Significant antecedents, including complex febrile seizures, meningitis, encephalitis, and trauma, occurred in $10(45 \%)$ patients. Mesial temporal sclerosis occurred in 12 of 15 with available hippocampal tissue, and cortical dysplasia of the temporal neocortex was found in 14 of 22 patients. These findings coexisted in 7 children. The high incidence of dual pathology may explain the early age of seizure onset.

\title{
DIAGNOSIS OF EPILEPSY FOLLOWING PAROXYSMAL EVENT
}

The accuracy of the initial diagnosis after one or more paroxysmal events is described as part of the Dutch Study of Epilepsy in Childhood (DSEC), a prospective hospital-based study of children with newly diagnosed possible single or multiple seizures. A panel of 3 pediatric neurologists classified events as epileptic seizures, unclear episodes, or events of definitely other origin. Children with unclear events were followed for 1 year and children with a diagnosis of seizures were followed for 2 years to assess the accuracy of the diagnosis. Single events in 224 children were classified initially as epileptic in $170(76 \%)$ and unclear in $54(24 \%)$. An epileptic diagnosis proved correct on follow-up in all 170 patients. Recurrent episodes permitted a definite epilepsy diagnosis in $4(7.4 \%)$ of the 54 children with unclear events. Multiple events in 536 children were classified initially as epilepsy in $412(77.7 \%$ ), and after follow-up the diagnosis was probably incorrect in $19(4.6 \%)$. In contrast, $7(5.6 \%)$ of 124 children with multiple episodes initially diagnosed as unclear were later classified as epilepsy. A false-positive diagnosis of epilepsy was made in $4.6 \%$, whereas a definite diagnosis of epilepsy was delayed in $5.6 \%$ of children with multiple unclear events and in $7.4 \%$ of children with a single unclear event. The sensitivity of the diagnosis of an epileptic seizure after a single paroxysmal event was $97.7 \%$ and the specificity $100 \%$; after multiple events, the epilepsy diagnosis sensitivity was $98.3 \%$ and specificity $86 \%$. Epileptiform abnormalities in the EEG confirm the diagnosis of epilepsy after multiple events in $90.1 \%$, and their absence negates the diagnosis in $46.9 \%$. (sensitivity, $70.3 \%$; specificity, $77.2 \%$ ). (Strink H, van Donselaar CA, Geerts AT et al. The accuracy of the diagnosis of paroxysmai events in chiidren. Neurology 25 March 2003;60:979-982). (Reprints: Dr H Stroink, Department of Neurology, St Elizabeth Hospital, PO Box 90151, 5000 LC Tilburg, the Netherlands).

COMMENT. The authors advise a conservative approach in children with paroxysmal events of uncertain nature. A false-negative diagnosis of epilepsy is considered less harmful for the patient than a false positive. It is of interest that in two events associated with teeth brushing and hair combing, despite an epileptiform EEG, a diagnosis of reflex epilepsy was rejected in favor of "hairdresser's syncope." 